\title{
Candidate Gene Identification of Feed Efficiency and Coat Color Traits in a C57BL/6J $\times$ Kunming F2 Mice Population Using Genome-Wide Association Study
}

\author{
Yuanxin Miao, ${ }^{1,2,3}$ Fathia Soudy, ${ }^{1,2}$ Zhong Xu, ${ }^{1,2}$ Mingxing Liao, ${ }^{1,2,4}$ \\ Shuhong Zhao, ${ }^{1,2}$ and ${\mathrm{Xinyun} \mathrm{Li}^{1,2}}^{1,2}$ \\ ${ }^{1}$ Key Laboratory of Agricultural Animal Genetics, Breeding and Reproduction of Ministry of Education and Key Laboratory of Swine \\ Genetics and Breeding of Ministry of Agriculture, Huazhong Agricultural University, Wuhan 430070, China \\ ${ }^{2}$ The Cooperative Innovation Center for Sustainable Pig Production, Wuhan 430070, China \\ ${ }^{3}$ Jingchu University of Technology, Jingmen 448000, China \\ ${ }^{4}$ Jingmen Animal Husbandry and Veterinary Bureau, Jingmen 448000, China
}

Correspondence should be addressed to Xinyun Li; xyli@mail.hzau.edu.cn

Received 1 May 2017; Accepted 21 June 2017; Published 30 July 2017

Academic Editor: Rituraj Purohit

Copyright (C) 2017 Yuanxin Miao et al. This is an open access article distributed under the Creative Commons Attribution License, which permits unrestricted use, distribution, and reproduction in any medium, provided the original work is properly cited.

\begin{abstract}
Feed efficiency (FE) is a very important trait in livestock industry. Identification of the candidate genes could be of benefit for the improvement of FE trait. Mouse is used as the model for many studies in mammals. In this study, the candidate genes related to FE and coat color were identified using C57BL/6J (C57) $\times$ Kunming (KM) F2 mouse population. GWAS results showed that 61 and 2 SNPs were genome-wise suggestive significantly associated with feed conversion ratio (FCR) and feed intake (FI) traits, respectively. Moreover, the Erbin, Msrb2, Ptfla, and Fgf10 were considered as the candidate genes of FE. The Lpl was considered as the candidate gene of FI. Further, the coat color trait was studied. KM mice are white and C57 ones are black. The GWAS results showed that the most significant SNP was located at chromosome 7, and the closely linked gene was Tyr. Therefore, our study offered useful target genes related to FE in mice; these genes may play similar roles in FE of livestock. Also, we identified the major gene of coat color in mice, which would be useful for better understanding of natural mutation of the coat color in mice.
\end{abstract}

\section{Introduction}

Feed efficiency (FE) is an important trait in livestock. In pigs, feed takes more than $60 \%$ of the total costs. Improvement of $\mathrm{FE}$ is one of the most important approaches for reducing the productive cost of livestock. At present, the assessment of FE is by using two highly correlated indicators: FCR (feed conversion ratio) and RFI (residual feed intake) [1,2], which lower FCR, and RFI means better FE [3-5].

Mice were widely used as animal model in energy metabolism, feed intake, and FE studies [6-8]. Recent study showed that oxygen consumption was negatively associated with FE of mice. Also, the higher oxygen consumption mice have higher mitochondrial activity and energy expenditure than lower oxygen consumption mice $[9,10]$. These results indicated that energy metabolism was negatively related to
FE in mice. Similarly, the energy metabolism pathway in the skeletal muscle tissue was found negatively related to FE in pigs [11, 12]. Further, lots of studies confirmed that the hypothalamus can regulate the food intake of mice [1315]. The Grelin, Leptin, MC4R, NPY, and Agrp genes have been confirmed to participate in the regulation of feed intake in mice through hypothalamus neurons [16-18]. Orally administered tributyltin (TBT) can significantly increase the FE through inhibiting feed intake in mice [19]. In pigs, feed intake was also negatively related to $\mathrm{FE}$ and the $M C 4 R$ has been confirmed as a candidate gene of FE in pigs [20-22]. These studies indicate that mice are the ideal model for FE study of livestock.

The molecular mechanisms of the FE trait of farm animals have been partially revealed in previous studies. It has been 
TABLE 1: The descriptive statistics of growth traits of F2 generation mice.

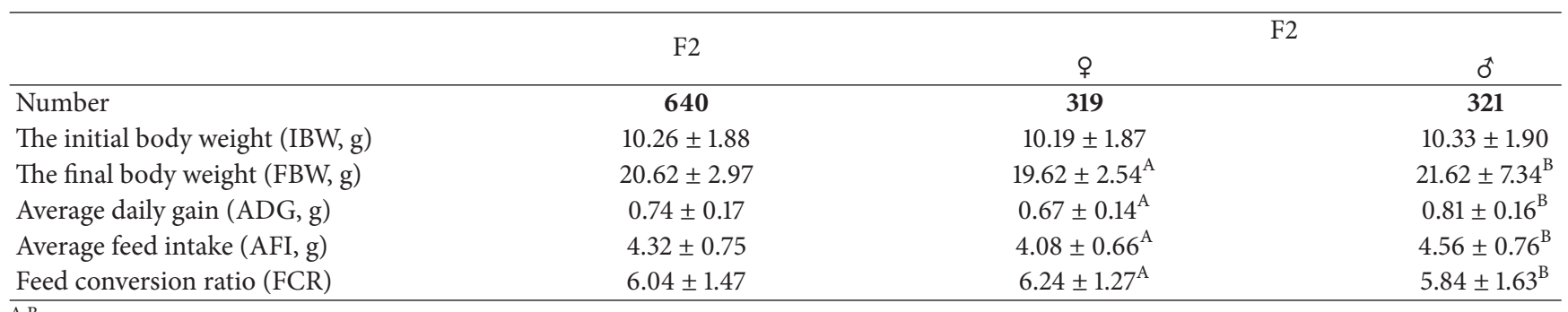

${ }_{\mathrm{A}, \mathrm{B}}$ Different letters within a row represent significant differences at $P<0.01$.

TABLE 2: The correlation analysis between the traits of F2 generation mice.

\begin{tabular}{|c|c|c|c|c|c|}
\hline & IBW & FBW & $\mathrm{ADG}$ & FI & FCR \\
\hline IBW & 1.00 & & & & \\
\hline FBW & 0.63 & 1.00 & & & \\
\hline ADG & -0.01 & 0.77 & 1.00 & & \\
\hline AFI & 0.33 & 0.72 & 0.65 & 1.00 & \\
\hline FCR & 0.27 & -0.30 & -0.60 & 0.11 & 1.00 \\
\hline
\end{tabular}

reported that genes involved in lipogenic and steroidogenic pathways were downregulated in the liver and fat tissues of high FE pigs. Further, signaling pathway analysis indicated that ESR1 could affect the FE trait via regulating acute caloric restriction of pigs [23]. Our former study showed that vitamin A metabolism pathway in liver tissue played important roles in regulation of FE in pigs [24]. Furthermore, FE trait could also be affected by immune response [25]. The genes that were expressed in hypothalamus and participated into lipid metabolism were also found to regulate FE in Angus cattle and chicks [26, 27].

Some candidate genes related to FE have been identified in previous studies. One study showed that GLP1R, CDKAL, and SGMS1genes, which related to insulin release, were associated with pig RFI and ADFI traits based on the GWAS results of 1,400 pigs [28]. Also, MAP3K5, PEX7, and DSCAM gene were suggested as candidate genes of RFI traits based on the GWAS results of 1,272 pigs [29]. In addition, HIF1AN on SSC4 and LBX1 on SSC14 have been speculated as possible candidate genes of FCR based on the GWAS results of 3,071 Duroc pigs [30]. Though some candidate genes of FE have been identified, few of them can be mutually authenticated in different studies, and the major genes of FE were still largely unknown.

In this study, the candidate genes related to $\mathrm{FE}$ have been identified using mouse model. According to the GWAS results, Erbin, Msrb2, Ptf1a, and Fgf10 genes closely linked with the top 3 significant SNPs were considered as candidate genes of FCR. The $L p l$ genes closely linked with the 2 genomewise suggestive significant SNPs of FI was considered as its candidate gene. Also, the Tyr gene was suggested as the major genes of coat color of mice.

\section{Results}

2.1. FCR and ADG Are High Correlated in C57 $\times$ KM F2 Population. The growth rate and FE traits were of significant difference between KM and C57 mice. KM mice growth was much faster and they have better FE compared to C57 mice at the age of 3 to 5 weeks. Therefore, a C $57 \times$ KM F2 segregation population has been constructed and the AFI, FCR, and ADG traits at 3 to 5 weeks of each of the $\mathrm{F} 2$ mice were measured. As a result, ADG of F2 mice is $0.74 \mathrm{~g} /$ day, AFI of F2 mice is $4.32 \mathrm{~g} /$ day, and FCR is 6.04. Statistic analysis indicated that the ADG, FI, and FCR traits were significantly different between males and females (Table 1). Male mice have better $\mathrm{FE}$ and higher AFI and growth rate than those of female mice. Correlation analysis indicated there were high correlations between ADG with final body weight (FBW) $(R=0.77)$, FI $(R=0.65)$, and FCR $(R=-0.6)$, while there was weak correlation between FI and FCR $(R=0.11)$ (Table 2).

2.2. The Results of RAD-Seq and SNP Calling. After the F2 population construction, 34 extremely high FCR and 38 extremely low FCR were selected for RAD-Seq. In the high FE group, FCR of females is $8.33 \pm 1.59$; and FCR of males is 7.81 \pm 1.45 . In the low FE group, FCR of females is $4.85 \pm 0.42$, and FCR of males is $4.76 \pm 0.53$ (Table 3 ). For the RAD-Seq, more than 500 million clean reads were yielded. The ratio of clean data in raw data were greater than $90 \%$, the error rates were less than $0.05 \%$, the Q20 and Q30 were greater than $90 \%$, and the GC contents were close to $40 \%$ (Table 4). After sequence, the SNPs were identified. As a result, 988,717 raw SNPs were firstly identified according to RAD-Seq.

2.3. GWAS Results of FCR and AFI Traits. In order to identify the candidate genes and SNPs of FE trait, GWAS was performed by using 92132 high quality SNPs. As a result, 61 SNPs associated with FCR traits were found at genome-wise suggestive significant level $(P<1)$. Among them, 2 SNPs were significantly associated with FCR traits $(P<0.05)$, located at chromosomes 13 and 2, respectively (Figure 1). The Erbin, Msrb2, Ptfla, and Fgf10 genes were closely linked with the 
TABLE 3: The descriptive statistics of growth traits for RAD-Seq individuals.

\begin{tabular}{|c|c|c|c|c|}
\hline & \multicolumn{2}{|c|}{ High FCR } & \multicolumn{2}{|c|}{ Low FCR } \\
\hline & 우 $(n=17)$ & $\widehat{\jmath}(n=17)$ & 우 $(n=21)$ & $\widehat{o}(n=17)$ \\
\hline IBW (g) & $9.51 \pm 1.67$ & $11.19 \pm 1.52$ & $8.53 \pm 1.55$ & $9.03 \pm 2.00$ \\
\hline FBW (g) & $24.73 \pm 10.50$ & $25.53 \pm 6.79$ & $24.05 \pm 7.35$ & $22.88 \pm 4.57$ \\
\hline $\mathrm{ADG}(\mathrm{g})$ & $0.52 \pm 0.15$ & $0.61 \pm 0.19$ & $0.80 \pm 0.13$ & $0.91 \pm 0.15$ \\
\hline AFI (g) & $4.16 \pm 0.87$ & $4.59 \pm 1.06$ & $3.85 \pm 0.56$ & $4.31 \pm 0.64$ \\
\hline FCR & $8.33 \pm 1.59$ & $7.81 \pm 1.45$ & $4.85 \pm 0.42$ & $4.76 \pm 0.53$ \\
\hline
\end{tabular}

TABLE 4: The sequence quality status of RAD-Seq data.

\begin{tabular}{lcc}
\hline & High FCR & Low FCR \\
\hline Raw base $\left(10^{8} \mathrm{bp}\right)$ & $18.79 \pm 14.54$ & $13.13 \pm 5.76$ \\
Clean base $\left(10^{8} \mathrm{bp}\right)$ & $17.67 \pm 13.81$ & $12.28 \pm 5.56$ \\
Effective rate $(\%)$ & $93.62 \pm 1.80$ & $93.04 \pm 1.72$ \\
Error rate $(\%)$ & $0.03 \pm 0.00$ & $0.03 \pm 0.00$ \\
Q20 $(\%)$ & $96.88 \pm 0.29$ & $96.61 \pm 0.58$ \\
Q30 $(\%)$ & $91.46 \pm 0.53$ & $90.78 \pm 1.36$ \\
GC content $(\%)$ & $40.21 \pm 0.62$ & $40.48 \pm 0.82$ \\
\hline
\end{tabular}

Effective rate (\%): the percentage of clean data in raw data; error rate (\%): base error rate; Q20 and Q30 (\%): the percentage of base with Phred value for greater than 20 or 30; GC content (\%): the percentage of $\mathrm{G}$ and $\mathrm{C}$ base.
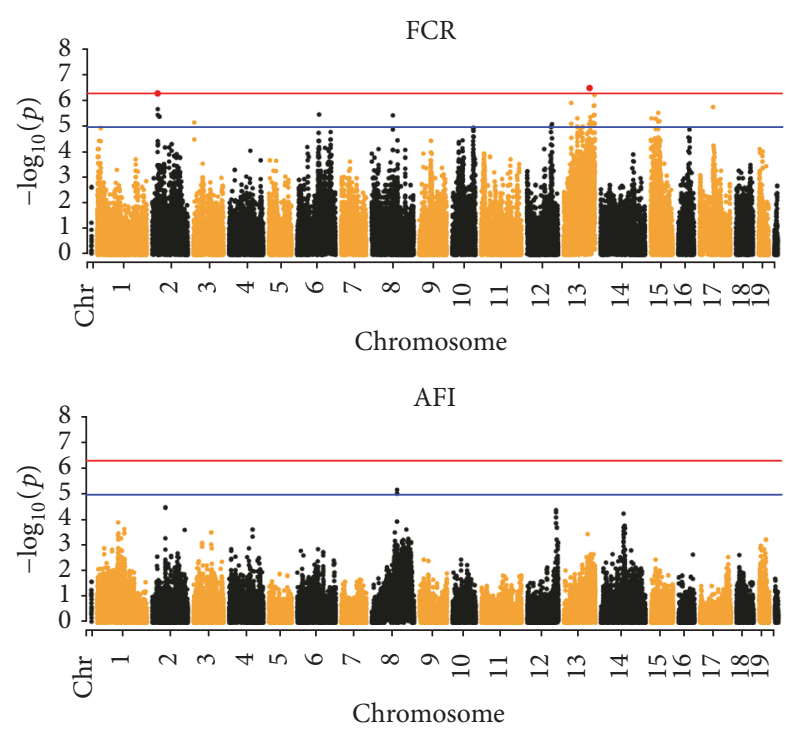

FIGURE 1: Manhattan plot of genome-wide association analysis studies for FCR and AFI. The red and blue lines indicate Bonferroni corrected $P=0.05$ and 1 , respectively.

top 3 SNPs of FCR (Table 5). Also, 2 SNPs associated with AFI were found at genome-wise suggestive significance $(P<$ 1) (Figure 1), which was closely linked with Lpl gene (Table 5).

2.4. GWAS Result Indicated That Tyr Gene Was the Candidate Gene of Coat Color. The coat color of KM mice is white and that of C57 mice is black. In the F2 population, there were 459 black and 181 white ones. The ratio of black: white is approximately $3: 1$. The 72 mice were chosen for RADSequencing, including 49 black and 23 white. The GWAS

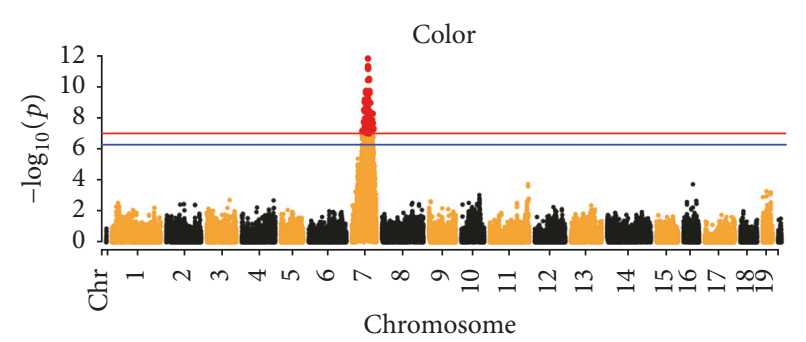

(a)

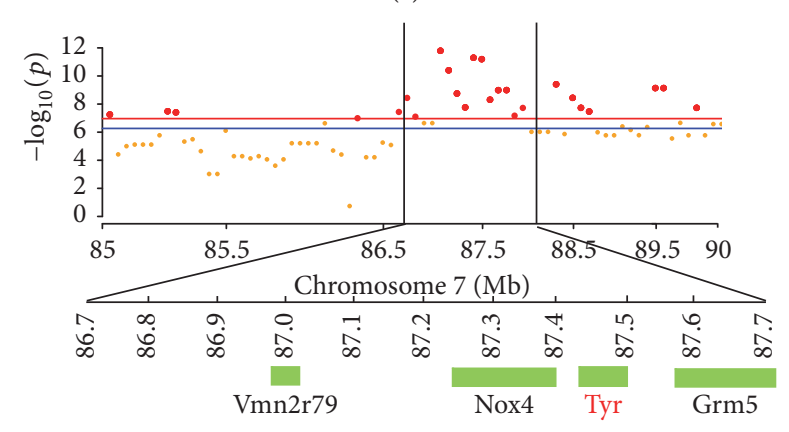

(b)

Figure 2: Manhattan plot and the candidate genes of coat color traits. The red and blue lines indicate Bonferroni corrected $P=0.01$ and 0.05 , respectively. Four genes were closely linked with the 13 most significant SNPs.

result showed there was strong signal on chromosome 7 (Figure 2). In total, 332 SNPs were significantly associated with the coat color at genome-wide level $(P<0.05)$ (Figure $2(\mathrm{a}))$. In the $87.16 \sim 87.68 \mathrm{Mb}$, there were 13 significant SNPs $(P<0.01)$. The most significant SNP was closely linked with Vmn2r79, Nox4, Tyr, and Grm5 genes (Figure 2). 
TABLE 5: The candidate genes in the regions of $0.5 \mathrm{Mb}$ nearby the suggestive significant SNPs for FCR and AFI.

\begin{tabular}{ccccc}
\hline Trait & CHR & SNP location & $P$ value & Adjacent genes $( \pm 0.5 \mathrm{Mb})$ \\
\hline \multirow{2}{*}{ FCR } & 13 & 103458907 & $3.36 E-07$ & Erbin \\
& 2 & 19827406 & $5.40 E-07$ & Msrb2 Ptfla \\
\hline \multirow{2}{*}{ AFI } & 13 & 118659655 & $6.23 E-07$ & Fgflo \\
& 8 & 69019203 & $6.82 E-06$ & $L p l$ \\
\hline
\end{tabular}

\section{Discussion}

Feed efficiency is an important economic trait in farm animals. Identification of the major genes would be useful for improving this trait. At present, although some signaling pathways have been suggested to be related to FE, not many candidate genes have been suggested. Moreover, few candidate genes have been validated. One possible reason is that FE is a complex economic trait, which may be regulated by many genes. The effect of each gene may not be very large. The second reason is that the population used for FE study may not be good enough. The sample size may not be big enough due to restriction of funding or labor. At present, the major genes of FCR were still not very clear. In this study, we adopt a new strategy. The two mouse strains with significant difference at FE traits were chosen, and the extremely high FE and low FE F2 mice were chosen for GWAS analysis. Some candidate genes of FE have been identified using the F2 mice population. As we know, mice are used as a model for many studies due to high conservation of genome between mammals. Therefore, these candidate genes could also be useful for the improvement of FE in farm animals.

In this study, four candidate genes of FCR have been identified. Functional study showed that Erbin could bind and segregate phosphorylated Smad2/3 complex, which blocked the TGF- $\beta$ signaling $[31,32]$. Also, the phosphorylation of Smad2/3 can transduce signals of MSTN, one of the most powerful inhibitors of muscle growth [33-35]. Moreover, Erbin has been reported to inhibit cardiac hypertrophy via ERK signaling pathway $[36,37]$. Therefore, we deduce that Erbin may regulate FE trait through affecting muscle growth especially via TGF- $\beta$ signaling pathway. Msrb2 is highly expressed in skeletal muscle tissue, which is an oxidoreductase in the mitochondria and responsible for elimination of intracellular reactive oxygen species (ROS) [38, 39]. Many studies indicated that ROS play important roles in muscle growth and increase ROS level accompanied with muscle mass loss [40-42]. Therefore, we deduced that Msrb2 may regulate FE through affecting the ROS level in the skeletal muscle tissue. Ptfla gene is the key factor for the fate determination of the pancreatic exocrine cells which is indispensable for pancreas development [43]. Ptfla-null mice were dead soon after birth, and inhibition of Ptfla resulted in pancreatic hypoplasia, glucose intolerance, and insufficient insulin secretion in a dosage-dependent manner [44]. Fgf10 has been reported to participate in adipogenesis. $\mathrm{Fg} f 10$ knock-out mice die shortly after birth, and the preadipocyte proliferation and adipogenesis are greatly impaired. Signaling pathway studies indicated that FGF10 could activate FGF receptor $2 \mathrm{~b}$
(FGFR2b) and stimulates preadipocyte proliferation and adipogenesis through the downstream Ras/MAPK/C/EBP $\alpha$ pathway [45]. Glucose and adipose metabolism are also important for the regulation of FE [12]. Therefore, Ptfla and FGF10 gene may participate into regulation of FE through affection glucose and adipose metabolism.

For feed intake trait, Lipoprotein lipase ( $L p l)$ was suggested as the candidate gene in this study. Previous studies indicated that $L p l$ is a multifunctional enzyme that plays major roles in the metabolism and transport of lipids in peripheral tissues [46, 47]. Muscle-specific Lpl transgenic mice showed TG accumulation and insulin resistance [48, 49]. Also, muscle-specific Lpl knock-out could increase the insulin mediated glucose uptake in the skeletal muscle tissue [50]. Lpl also presents in brain; specific mice with knockout of $L p l$ in neuron (NEXLPL-/-) are hyperphagic and obesity. Functional study showed that AgRp and Mc3r were significantly upregulated in the neuron specific $L p l$ knock-out mice [51]. These studies indicated that Lpl played important roles in energy metabolism, which could affect feed intake, glucose, and lipids metabolism in mice. Thus, we conclude that $L p l$ may be candidate gene of AFI of mice.

The candidate gene for the coat color has also been analyzed. The genotypes of the mice have been detected using the top significant SNP. The heterozygosity/homozygosity ratios in the high FE group (AA: $\mathrm{AC}: \mathrm{CC}=5: 18: 11$ ) and low FE group (AA : $\mathrm{AC}: \mathrm{CC}=5: 20: 13$ ) have no significant difference. This indicated that the coat color trait was not correlated with the FE trait. According to the GWAS results, the Tyr gene has been considered as the major gene of coat color trait. Tyr is known to be the rate-limiting enzyme affecting the production of melanin pigment [52], which oxidates tyrosine to dihydroxyphenylalanine (DOPA) and determines which type of melanin could be synthesized [53]. Previous studies have shown that mutations of the Tyr gene are associated with albinism phenotype of mouse [54], human [55], cattle [56], and rat [57]. Therefore, we conclude Tyr is the major gene of coat color trait.

\section{Materials and Methods}

4.1. Population Constructed. In this study, a F2 segregation population of C57BL/6 (C57) $\times$ Kunming (KM) mice was constructed. C57 mice are black, which grow slow and have low FE. KM mice are white, which grow fast and have high FE. Thus, 7 KM females and 7 C57 males were chosen as the founder for F2 population construction. In total, $640 \mathrm{~F} 2$ mice including 319 females and 321 males were generated. 
All mice were housed under controlled temperature $(21 \pm$ $2^{\circ} \mathrm{C}$ ) on a $12: 12$ hour light-dark cycle with free access to food and water. The body weight at 3 weeks and 5 weeks and the total feed intake of this period of each of the F2 mice were measured. Furthermore, average day gain (ADG), average day feed intake (AFI), and feed conversion ratio (FCR) were measured. All the methods in this study were carried out in accordance with the approved guidelines from the Regulation of the Standing Committee of Hubei People's Congress. Furthermore, all experimental protocols were approved by the Ethics Committee of Huazhong Agricultural University (HZAUMU2013-0005).

4.2. DNA Preparation and RAD-Sequencing. The tail samples of the F2 mice were collected at 5 weeks of age. Total DNA of mouse tail was extracted with E-Z $96^{\circledR}$ Mag-Bind ${ }^{\circledR}$ Tissue DNA kit (omega, USA), according to the manufacturer's instructions. Gel electrophoresis and NanoDrop ND2000 spectrophotometry (Thermo Fisher Scientific, USA) were used to detect the quality and concentration of DNA. Then 34 extremely high and 38 extremely low FCR F2 mice were chosen for RAD-Sequencing. RAD-Sequencing was performed by a commercial company service (Novogene, Beijing, China). In brief, mice DNA samples were firstly digested by using EcoR I restriction enzymes. Then, the P1 and P2 adapters were ligated onto the DNA fragments. The P1 and P2 adaptor contains the Illumina PCR Forward and Reverse primer sequences. After 12-cycle PCR amplification, the 350 550 bp DNA fragments were collected and sequenced by using Illumina HiSeq2000.

4.3. SNP Mining and GWAS Analysis. The reads quality of the raw data was first evaluated and the low quality reads were filtered according to the criteria $(N>10 \%$; $Q<=$ $5)$. Then, the adapter sequence of the high quality reads were trimmed, which were named as clean reads. The clean reads were then mapped to mouse genome (Version number GRCm38.75) using BWA (Burrows-Wheeler Aligner) software. The PCR redundancy was removed by using Picardtools. The unique mapping reads with less than 3 mismatch Nt were chosen for SNP calling. The SNPs were called by using GATK software under the criteria (Qual score $\geq 30$, $\mathrm{QD}<20.0$, ReadPosRankSum $<-8.0$, FS $>10.0$ and QUAL $<$ \$MEANQUA). Those SNPs with the criteria calling rate $\geq 70 \%$, MAF $\geq 1 \%$, and HWE $P \geq 10^{-6}$ were identified. Subsequently, the missing SNPs were amputated by using Beagle Genetic Analysis software. GWAS analysis was finally performed using the 92132 SNPs and 72 samples with the criteria $\mathrm{MAF} \geq 5 \%$, genotyping call rate $\geq 95 \%$, and $\mathrm{HWE}$ $P \geq 10^{-6}$. The significant SNPs were analyzed by using Plink software based on case/control model. Bonferroni corrected $P$ values were adopted for the genome-wide significance threshold that was set as $1 / N, 0.05 / N$, and $0.01 / N$, three levels, where $N$ is the number of total SNPs used for GWAS.

4.4. Statistic Analysis. The differences of the traits between male and female mice were analyzed by analyses of variance using SAS. Pearson's correlation coefficients of difference in traits were counted by using SAS.

\section{Conclusions}

In conclusion, we constructed a C57 $\times$ KM F2 segregation population. The results of GWAS indicated that the Erbin, Msrb2, Ptfla, and Fgf10 genes could be candidate genes of FCR in mice. Lpl could be candidate gene of AFI in mice. These genes may also be the candidate genes of the FCR and AFI traits in livestock. Also, Tyr is considered as the candidate gene of coat color in mice. This study offered new candidate genes for FE, which would be useful for bettered understanding of the mechanisms of FE trait. Also, the major gene of coat color offered new evidence of genetic variation of this trait in mice.

\section{Conflicts of Interest}

The authors declare no conflicts of interest.

\section{Authors' Contributions}

Shuhong Zhao and Xinyun Li conceived and designed the experiments; Yuanxin Miao, Zhong Xu, Mingxing Liao, and Fathia Soudy performed the experiments; Yuanxin Miao analyzed the data; Yuanxin Miao and Fathia Soudy contributed reagents/materials/analysis tools; Yuanxin Miao, Xinyun Li, and Shuhong Zhao wrote the manuscript. Yuanxin Miao and Fathia Soudy contributed equally to this work.

\section{Acknowledgments}

This research was supported by the National Natural Science Foundation of China (31372291), the National High Technology Research and Development Program of China (863 Program, 2013AA102502), the Natural Science Foundation of Hubei Provincial Department of education (Q20164305), and the Jingchu University of Technology (QDB201607).

\section{References}

[1] D. H. Jr Crews, "Genetics of efficient feed utilization and national cattle evaluation: a review," Genetics and Molecular Research, vol. 4, pp. 152-165, 2005.

[2] J. M. Young, W. Cai, and J. C. M. Dekkers, "Effect of selection for residual feed intake on feeding behavior and daily feed intake patterns in yorkshire swine," Journal of Animal Science, vol. 89, no. 3, pp. 639-647, 2011.

[3] R. Saintilan, I. Mérour, L. Brossard et al., "Genetics of residual feed intake in growing pigs: Relationships with production traits, and nitrogen and phosphorus excretion traits," Journal of Animal Science, vol. 91, no. 6, pp. 2542-2554, 2013.

[4] D. N. Do, A. B. Strathe, J. Jensen, T. Mark, and H. N. Kadarmideen, "Genetic parameters for different measures of feed efficiency and related traits in boars of three pig breeds," Journal of Animal Science, vol. 91, no. 9, pp. 4069-4079, 2013.

[5] R. Barea, S. Dubois, H. Gilbert, P. Sellier, J. van Milgen, and J. Noblet, "Energy utilization in pigs selected for high and low 
residual feed intake," Journal of Animal Science, vol. 88, no. 6, pp. 2062-2072, 2010.

[6] J. Lu, Q. Xu, M. Ji et al., "The phosphorylation status of t522 modulates tissue-specific functions of sirtl in energy metabolism in mice," EMBO Reports, 2017.

[7] J. D. Douglass, Y. X. Zhou, A. Wu et al., "Global deletion of MGL in mice delays lipid absorption and alters energy homeostasis and diet-induced obesity," Journal of Lipid Research, vol. 56, no. 6, pp. 1153-1171, 2015.

[8] P. A. Sojka, R. S. Griess, and M. K. Nielsen, "Locomotor activity and body temperature in selected mouse lines differing greatly in feed intake," Journal of Animal Science, vol. 91, no. 8, pp. 35573563, 2013.

[9] H. Darhan, M. Kikusato, M. Toyomizu et al., "Selection for high and low oxygen consumption-induced differences in maintenance energy requirements of mice," Animal Science Journal, 2016.

[10] Y. Hong, A. Ardiyanti, M. Kikusato, T. Shimazu, M. Toyomizu, and K. Suzuki, "Selection for high and low oxygen consumption altered hepatic mitochondrial energy efficiency in mice," Animal Science Journal, vol. 86, no. 9, pp. 818-825, 2015.

[11] L. Jing, Y. Hou, H. Wu et al., “Transcriptome analysis of mRNA and miRNA in skeletal muscle indicates an important network for differential Residual Feed Intake in pigs," Scientific Reports, vol. 5, Article ID 11953, 2015.

[12] F. Liangliang, X. Yueyuan, Y. Hou et al., "Proteomic analysis indicates that mitochondrial energy metabolism in skeletal muscle tissue is negatively correlated with feed efficiency in pigs," Scientific Reports, 2017.

[13] Y.-C. Shi, Z. Lin, J. Lau et al., "PYY3-36 and pancreatic polypeptide reduce food intake in an additive manner via distinct hypothalamic dependent pathways in mice," Obesity, vol. 21, no. 12, pp. E669-E678, 2013.

[14] A. Caron, S. M. Labbé, D. Lanfray et al., "Mediobasal hypothalamic overexpression of DEPTOR protects against high-fat dietinduced obesity," Molecular Metabolism, vol. 5, no. 2, pp. 102112, 2016.

[15] A. Valle, N. Hoggard, A. C. Adams, P. Roca, and J. R. Speakman, "Chronic central administration of apelin-13 over 10 days increases food intake, body weight, locomotor activity and body temperature in C57BL/6 mice," Journal of Neuroendocrinology, vol. 20, no. 1, pp. 79-84, 2008.

[16] J. S. Zakhari, E. P. Zorrilla, B. Zhou, A. V. Mayorov, and K. D. Janda, "Oligoclonal antibody targeting ghrelin increases energy expenditure and reduces food intake in fasted mice," Molecular Pharmaceutics, vol. 9, no. 2, pp. 281-289, 2012.

[17] E. Schéle, T. Bake, C. Rabasa, and S. L. Dickson, "Centrally administered ghrelin acutely influences food choice in rodents," PLoS ONE, vol. 11, no. 2, Article ID e0149456, 2016.

[18] C. G. Wagner, C. D. McMahon, D. L. Marks, J. A. Daniel, B. Steele, and J. L. Sartin, "A role for agouti-related protein in appetite regulation in a species with continuous nutrient delivery," Neuroendocrinology, vol. 80, no. 4, pp. 210-218, 2004.

[19] E. Bo, A. Farinetti, M. Marraudino et al., "Adult exposure to tributyltin affects hypothalamic neuropeptide Y, Y1 receptor distribution, and circulating leptin in mice," Andrology, vol. 4, no. 4, pp. 723-734, 2016.

[20] B. Fan, S. Lkhagvadorj, W. Cai et al., "Identification of genetic markers associated with residual feed intake and meat quality traits in the pig," Meat Science, vol. 84, no. 4, pp. 645-650, 2010.
[21] K. S. Kim, N. Larsen, T. Short, G. Plastow, and M. F. Rothschild, "A missense variant of the porcine melanocortin-4 receptor (MC4R) gene is associated with fatness, growth, and feed intake traits," Mammalian Genome, vol. 11, no. 2, pp. 131-135, 2000.

[22] R. Davoli, S. Braglia, V. Valastro et al., "Analysis of MC4R polymorphism in Italian Large White and Italian Duroc pigs: Association with carcass traits," Meat Science, vol. 90, no. 4, pp. 887-892, 2012.

[23] S. Lkhagvadorj, L. Qu, W. Cai et al., "Gene expression profiling of the short-term adaptive response to acute caloric restriction in liver and adipose tissues of pigs differing in feed efficiency," American Journal of Physiology - Regulatory Integrative and Comparative Physiology, vol. 298, no. 2, pp. R494-R507, 2010.

[24] Y. Zhao, Y. Hou, F. Liu et al., “Transcriptome analysis reveals that vitamin a metabolism in the liver affects feed efficiency in pigs," G3: Genes, Genomes, Genetics, vol. 6, no. 11, pp. 3615-3624, 2016.

[25] M. Jégou, F. Gondret, A. Vincent, C. Tréfeu, H. Gilbert, and I. Louveau, "Whole blood transcriptomics is relevant to identify molecular changes in response to genetic selection for feed efficiency and nutritional status in the pig," PLoS ONE, vol. 11, no. 1, Article ID e0146550, 2016.

[26] S. D. Perkins, C. N. Key, C. F. Garrett et al., "Residual feed intake studies in Angus-sired cattle reveal a potential role for hypothalamic gene expression in regulating feed efficiency," Journal of Animal Science, vol. 92, no. 2, pp. 549-560, 2014.

[27] E. L. Sherman, J. D. Nkrumah, B. M. Murdoch et al., "Polymorphisms and haplotypes in the bovine neuropeptide $\mathrm{Y}$, growth hormone receptor, ghrelin, insulin-like growth factor 2 , and uncoupling proteins 2 and 3 genes and their associations with measures of growth, performance, feed efficiency, and carcass merit in beef cattle," Journal of Animal Science, vol. 86, no. 1, pp. 1-16, 2008.

[28] S. K. Onteru, D. M. Gorbach, J. M. Young, D. J. Garrick, J. C. M. Dekkers, and M. F. Rothschild, "Whole Genome Association Studies of Residual Feed Intake and Related Traits in the Pig," PLoS ONE, vol. 8, no. 6, Article ID e61756, 2013.

[29] D. N. Do, T. Ostersen, A. B. Strathe, T. Mark, J. Jensen, and H. N. Kadarmideen, "Genome-wide association and systems genetic analyses of residual feed intake, daily feed consumption, backfat and weight gain in pigs," BMC Genetics, vol. 15, article no. 27, 2014.

[30] G. Sahana, V. Kadlecová, H. Hornshøj, B. Nielsen, and O. F. Christensen, "A genome-wide association scan in pig identifi es novel regions associated with feed effi ciency trait," Journal of Animal Science, vol. 91, no. 3, pp. 1041-1050, 2013.

[31] F. Dai, C. Chang, X. Lin, P. Dai, L. Mei, and X. H. Feng, "Erbin inhibits transforming growth factor beta signaling through a novel smad-interacting domain," Molecular \& Cellular Biology, vol. 27, pp. 6183-6194, 2007.

[32] G. Sflomos, E. Kostaras, E. Panopoulou et al., "ERBIN is a new SARA-interacting protein: Competition between SARA and SMAD2 and SMAD3 for binding to ERBIN," Journal of Cell Science, vol. 124, no. 19, pp. 3209-3222, 2011.

[33] L. Bradley, P. J. Yaworsky, and F. S. Walsh, "Myostatin as a therapeutic target for musculoskeletal disease," Cellular \& Molecular Life Sciences Cmls, vol. 65, pp. 2119-2124, 2008.

[34] K. Tsuchida, "Myostatin inhibition by a follistatin-derived peptide ameliorates the pathophysiology of muscular dystrophy model mice," Acta myologica: myopathies and cardiomyopathies: official journal of the Mediterranean Society of Myology / edited 
by the Gaetano Conte Academy for the study of striated muscle diseases, vol. 27, pp. 14-18, 2008.

[35] S. Bogdanovich, T. O. B. Krag, E. R. Barton et al., "Functional improvement of dystrophic muscle by myostatin blockade," Nature, vol. 420, no. 6914, pp. 418-421, 2002.

[36] I. Rachmin, S. Tshori, Y. Smith et al., "Erbin is a negative modulator of cardiac hypertrophy," Proceedings of the National Academy of Sciences of the United States of America, vol. 111, no. 16, pp. 5902-5907, 2014.

[37] I. Rachmin, E. Amsalem, E. Golomb et al., "FHL2 switches MITF from activator to repressor of Erbin expression during cardiac hypertrophy," International Journal of Cardiology, vol. 195, pp. 85-94, 2015.

[38] K. U. Schallreuter, K. Rübsam, B. Chavan et al., "Functioning methionine sulfoxide reductases $\mathrm{a}$ and $\mathrm{b}$ are present in human epidermal melanocytes in the cytosol and in the nucleus," Biochemical and Biophysical Research Communications, vol. 342, pp. 145-152, 2006.

[39] I. Pascual, I. M. Larrayoz, M. M. Campos, and I. R. Rodriguez, "Methionine sulfoxide reductase B2 is highly expressed in the retina and protects retinal pigmented epithelium cells from oxidative damage," Experimental Eye Research, vol. 90, no. 3, pp. 420-428, 2010.

[40] A. Espinosa, C. Henríquez-Olguín, and E. Jaimovich, "Reactive oxygen species and calcium signals in skeletal muscle: A crosstalk involved in both normal signaling and disease," Cell Calcium, 2016.

[41] K. P. Lee, Y. J. Shin, S. C. Cho et al., "Peroxiredoxin 3 has a crucial role in the contractile function of skeletal muscle by regulating mitochondrial homeostasis," Free Radical Biology \& Medicine, vol. 77, pp. 298-306, 2014.

[42] S. Sriram, S. Subramanian, P. K. Juvvuna et al., "Myostatin augments muscle-specific ring finger protein-1 expression through an NF-kB independent mechanism in SMAD3 null muscle," Molecular Endocrinology, vol. 28, no. 3, pp. 317-330, 2014.

[43] Y. Kawaguchi, B. Cooper, M. Gannon, M. Ray, R. J. MacDonald, and C. V. E. Wright, "The role of the transcriptional regulator Ptfla in converting intestinal to pancreatic progenitors," Nature Genetics, vol. 32, no. 1, pp. 128-134, 2002.

[44] A. Fukuda, Y. Kawaguchi, K. Furuyama et al., "Reduction of Ptfla gene dosage causes pancreatic hypoplasia and diabetes in mice," Diabetes, vol. 57, no. 9, pp. 2421-2431, 2008.

[45] H. Ohta, M. Konishi, and N. Itoh, "Fgf10 and fgf21 as regulators in adipocyte development and metabolism," Endocrine, Metabolic \& Immune Disorders-Drug Targets, vol. 11, pp. 302309, 2011.

[46] I. J. Goldberg, D. R. Soprano, M. L. Wyatt, T. M. Vanni, T. G. Kirchgessner, and M. C. Schotz, "Localization of lipoprotein lipase mrna in selected rat tissues," Journal of Lipid Research, vol. 30, pp. 1569-1577, 1989.

[47] H. Wang and R. H. Eckel, "Lipoprotein lipase: from gene to obesity," The American Journal of Physiology-Endocrinology and Metabolism, vol. 297, no. 2, pp. E271-E288, 2009.

[48] L. D. Ferreira, L. K. Pulawa, D. R. Jensen, and R. H. Eckel, "Overexpressing human lipoprotein lipase in mouse skeletal muscle is associated with insulin resistance," Diabetes, vol. 50, p. 1064, 2001.

[49] J. K. Kim, J. J. Fillmore, Y. Chen et al., “Tissue-specific overexpression of lipoprotein lipase causes tissue-specific insulin resistance," Proceedings of the National Academy of Sciences of the United States of America, vol. 98, no. 13, pp. 7522-7527, 2001.
[50] H. Wang, L. A. Knaub, D. R. Jensen et al., "Skeletal musclespecific deletion of lipoprotein lipase enhances insulin signaling in skeletal muscle but causes insulin resistance in liver and other tissues," Diabetes, vol. 58, no. 1, pp. 116-124, 2009.

[51] H. Wang, G. Astarita, M. D. Taussig et al., "Deficiency of lipoprotein lipase in neurons modifies the regulation of energy balance and leads to obesity," Cell Metabolism, vol. 13, no. 1, pp. 105-113, 2011.

[52] A. Sanchez-Ferrer, J. N. Rodriguez-Lopez, F. Garcia-Canovas, and F. Garcia-Carmona, "Tyrosinase: a comprehensive review of its mechanism," Biochim Biophys Acta, vol. 1247, pp. 1-11, 1995.

[53] S. Ito, K. Wakamatsu, and H. Ozeki, "Chemical analysis of melanins and its application to the study of the regulation of melanogenesis," Pigment Cell Research, vol. 13, no. 8, pp. 103$109,2000$.

[54] T. Yokoyama, D. W. Silversides, K. G. Waymire, B. S. Kwon, T. Takeuchi, and P. A. Overbeek, "Conserved cysteine to serine mutation in tyrosinase is responsible for the classical albino mutation in laboratory mice," Nucleic Acids Research, vol. 18, no. 24, pp. 7293-7298, 1990.

[55] W. S. Oetting, "The tyrosinase gene and oculocutaneous albinism type 1 (OCA1): a model for understanding the molecular biology of melanin formation," Pigment Cell Research, vol. 13 , no. 5, pp. 320-325, 2000.

[56] S. M. Schmutz, T. G. Berryere, D. C. Ciobanu, A. J. Mileham, B. H. Schmidtz, and M. Fredholm, "A form of albinism in cattle is caused by a tyrosinase frameshift mutation," Mammalian Genome, vol. 15, no. 1, pp. 62-67, 2004.

[57] W. M. Blaszczyk, L. Arning, K.-P. Hoffmann, and J. T. Epplen, "A Tyrosinase missense mutation causes albinism in the Wistar rat," Pigment Cell Research, vol. 18, no. 2, pp. 144-145, 2005. 

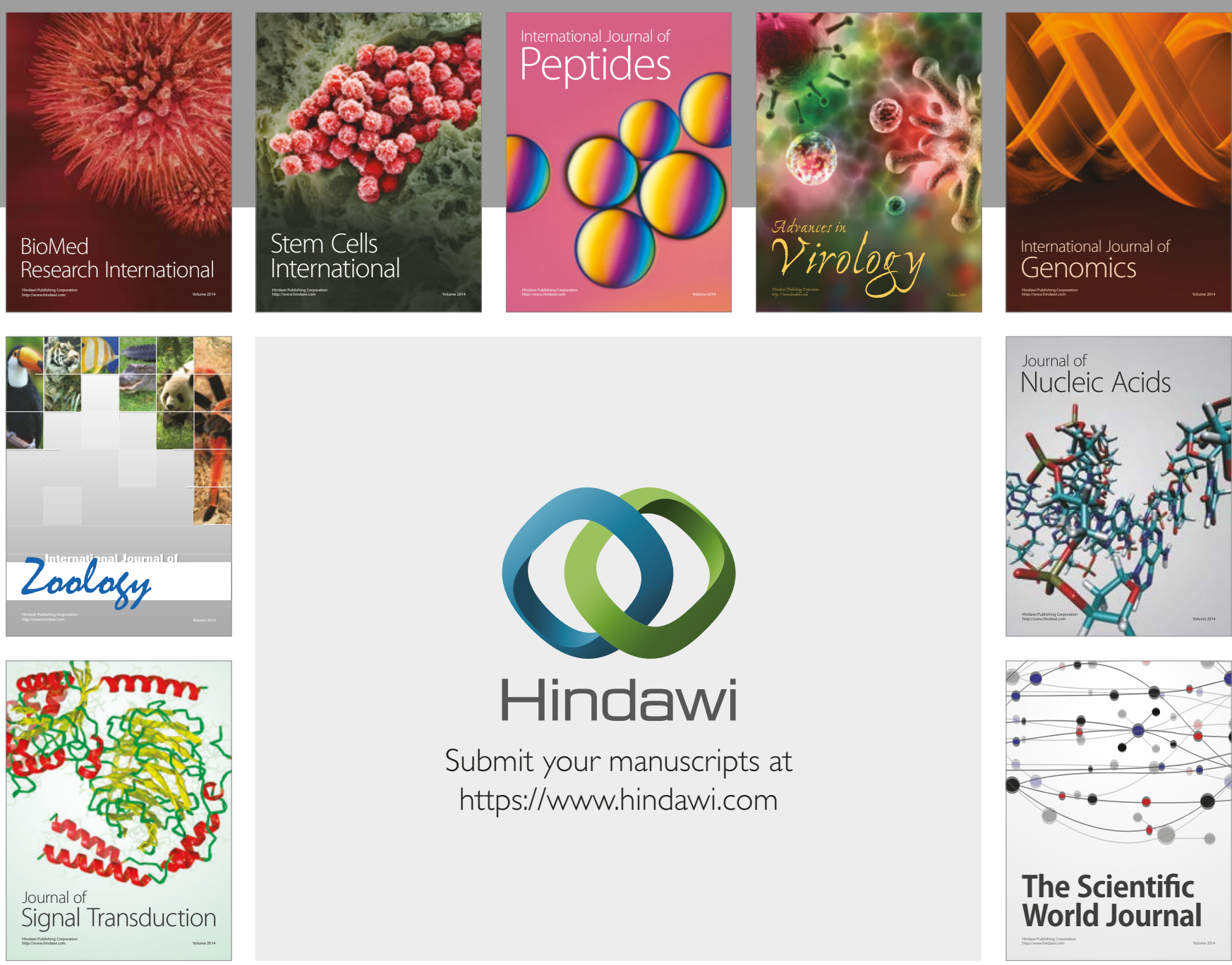

Submit your manuscripts at

https://www.hindawi.com
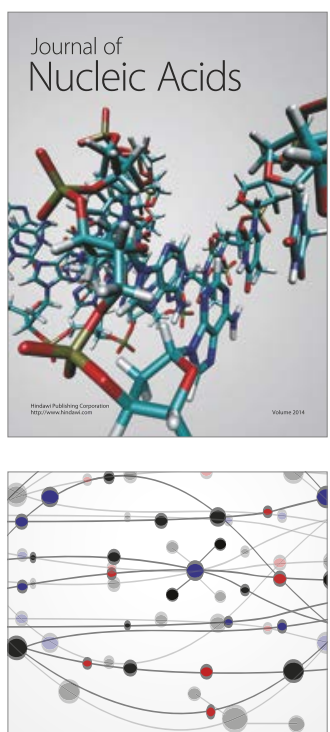

The Scientific World Journal

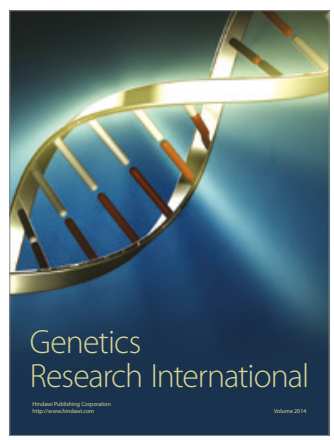

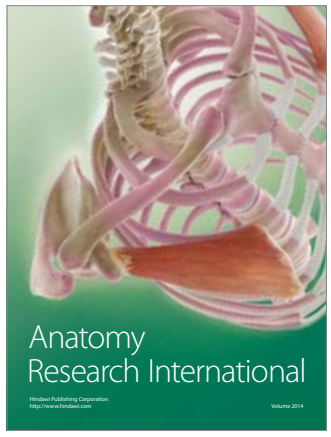

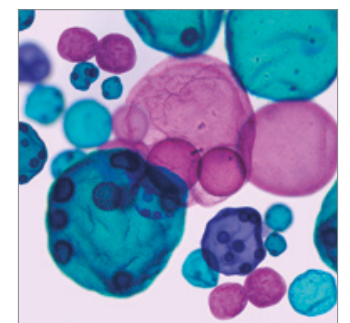

International Journal of Microbiology
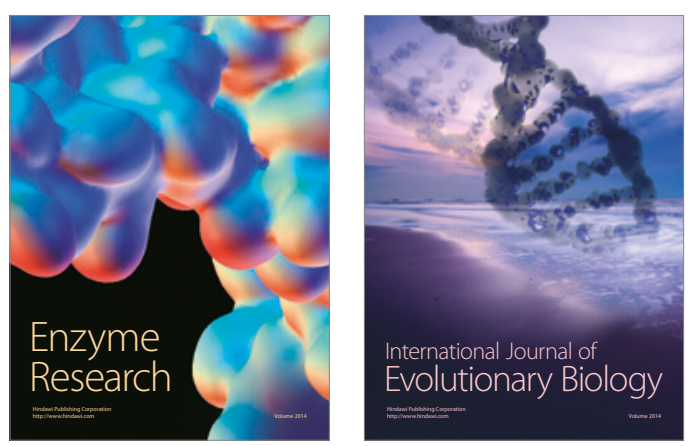
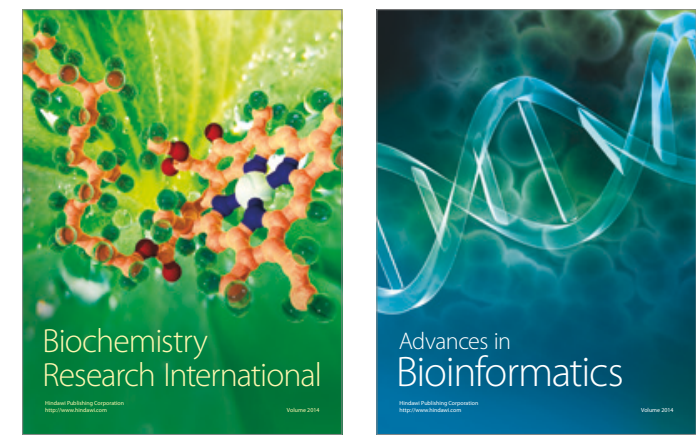

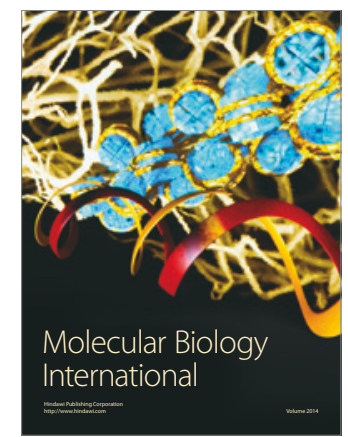

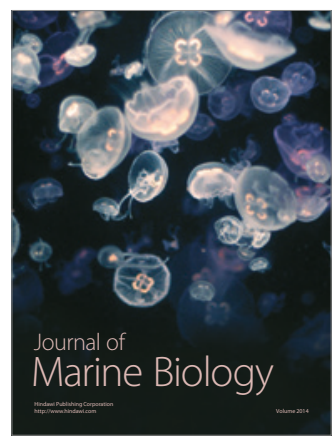

